\title{
ALGUNOS ASPECTOS TEÓRICOS SOBRE LA DISTRIBUCIÓN DEL INGRESO
}

\author{
NoRA Lustig \\ El Colegio de México
}

\section{INTRODUCCIÓN}

EL INTERÉs de este trabajo es: primero, ordenar algunas ideas sobre el tema de la distribución personal del ingreso en relación con algunos conceptos manejados en la teoría económica tales como el bienestar y la eficiencia; segundo, discutir con brevedad cómo la teoría económica explica (o más bien no explica), la formación de la distribución personal del ingreso, y por último, hacer una incursión somera en algunos enfoques que tratan de desarrollar un planteamiento teórico que explique el comportamiento de la misma.

\section{Distribución DEL INGRESO Y BIENESTAR}

La percepción de la desigualdad y su evaluación ética varía mucho entre las personas, las épocas y los lugares. De cualquier manera se podría hacer una división más o menos brusca entre aquellos que opinan, o han opinado, que la desigualdad es un fenómeno "natural": los seres humanos están dotados de diferentes dosis de inteligencia, belleza, habilidad física, etc., por lo tanto, es también "natural" que sea desigual la dotación de recursos económicos y la posibilidad de gozar de los beneficios que de ellos se deriven, y aquellos para quienes este hecho no es razón para perpetuar la injusticia en lo que atañe a las organizaciones sociales.

Cuando se habla de desigualdad económica es porque generalmente se tiene en mente una distribución "apropiada" del ingreso. O sea que la evaluación de desigualdad es relativa y depende de cómo la distribución que observemos discrepa de la que creemos es la "apropiada". Pero existen dos nociones que se disputan el honor de definir cuál distribución es la "apropiada": el principio de necesidad (versus) el principio del mérito, y nuestra noción de lo "justo" va a estar influida por uno o por ambos principios. Por ejemplo, si tomamos a dos individuos, uno de los cuales goza de perfecta salud y otro que sufre de alguna enfermedad crónica, las necesidades de este último van a ser mayores que las del primero (si suponemos que las demás características de ambos son similares). Con 
base en el principio de necesidad, el enfermo debería recibir más ingreso que la persona sana; en cambio, si nos guiáramos por el principio de méritos en relación con la productividad, es posible que le correspondería menos al enfermo.

Lo anterior tiene importancia en relación con el principio que favorece 1a igualdad total de la distribución basado en el supuesto de que las funciones de bienestar individuales son iguales para todos. Hemos visto cómo esta posición puede dar lugar a una distribución "indeseada" bajo el principio de necesidad, ya que un mismo nivel de ingreso puede no proveer del mismo bienestar a todos indistintamente.

La teoría del bienestar económico y la desigualdad en la distribución del ingreso. Por desgracia la teoría del bienestar econónico sirve poco para analizar los problemas de la desigualdad, dado que, en general, se concentra en análisis donde se evita el conflicto entre individuos. La concepción paretiana del bienestar implica una aceptación callada del statu quo. Según Pareto sólo se puede establecer que existe una mejora en el nivel de bienestar de la sociedad, cuando un cambio introducido mejora, por lo menos, la situación de uno de los individuos pero sin empeorar la de los demás. Este enfoque paretiano deja muy pocos grados de libertad para actuar en favor de una mejor distribución: si no se puede mejorar la situación del pobre quitándole al rico porque esta política implicaría un empeoramiento para el último, nos tenemos que contentar con una distribución "óptima" desde el punto de vista paretiano aunque fuera desigual.

Sin embargo, un elemento interesante se puede desprender de uno de los teoremas fundamentales de la teoría del bienestar: el de la equivalencia entre un equilibrio competitivo y un óptimo de Pareto, y la correspondencia que existe entre un equilibrio competitivo y la maximización de una función social de bienestar. ${ }^{1}$ Se sabe que el equilibrio competitivo implica un vector de cantidades y precios a que éstas deben intercambiarse para que haya equilibrio en cada mercado. El marco analítico del equilibrio supone una distribución inicial como parámetro; por lo tanto, puede haber tantas soluciones diferentes de equilibrio como distribuciones iniciales. Esto implica que los tipos de bienes, las cantidades y sus precios dependerán en parte de la distribución inicial del ingreso; más aún, se puede averiguar, cualitativamente al menos, la naturaleza del efecto de ésta con la introducción de una función de bienestar social que correspondiera al equilibrio competitivo. Se puede demostrar que esta función de bienestar social tiene la siguiente forma:

$$
\operatorname{Max} V\left(\ddot{x}_{i}\right):=\operatorname{Max} \Sigma_{a_{i}} u_{i}\left(x_{i}\right)
$$

1 T. Negishi, "Welfare Economics and Existence of an Equilibrium for a Competitive Economy", Universidad de Tokyo. 
donde $x_{i}$ son bienes y servicios, $u_{i}\left(x_{i}\right)$ son las funciones de utilidad individuales y $a_{i}$ las ponderaciones atribuidas a cada función de utilidad individual en la función de bienestar para toda la sociedad. ${ }^{2}$ Puede probarse que dichas ponderaciones son iguales al recíproco de la utilidad marginal del ingreso y, dado el supuesto de concavidad de la función individual de utilidad, se puede afirmar que el valor de $a_{i}$ será mayor cuando nos referimos a un individuo que posee más ingreso. Esto significa que la función de bienestar social que corresponde al equilibrio competitivo da mayor peso a las funciones de utilidad individual a medida que el ingreso del individuo aumenta. En otras palabras, el equilibrio competitivo está sesgado en favor de aquellos con mayor ingreso. Esta situación se puede comparar con la de una votación en la que algunas personas tienen derecho a más votos que otras, y en este caso particular los votos están dados por el poder adquisitivo que tenga cada individuo. Los precios resultantes del equilibrio competitivo, incluido el precio de los factores, son una media ponderada de los valores que los consumidores asignen a los bienes y servicios, y dichas ponderaciones dependerán de la distribución inicial.

De todo el análisis previo se pueden desprender dos conclusiones pertinentes al tema de la distribución dentro del contexto de la teoría del bienestar:

1) La distribución (funcional y personal) resultante del equilibrio depende de la distribución inicial, ya que por una parte ésta establece la cantidad de recursos que posee cada individuo y por otra influye en la determinación de los precios relativos a los que dichos recursos se van a evaluar; este resultado se puede generalizar a casos de mercados no competitivos, con la diferencia de que la influencia sobre la determinación de los precios por parte de los consumidores se verá disminuida por el poder que puedan ejercer sobre la formación de precios las empresas oligopólicas. De cualquier manera se podría afirmar que la estructura productiva y la retribución siguen siendo influenciadas por la distribución. (Las empresas oligopólicas producirán bienes para aquellos que pueden adquirirlos.)

2) Otra conclusión importante es que dado que los precios resultantes de un equilibrio competitivo varían de acuerdo con la distribución inicial, así como también cambia la estructura productiva (los bienes que se producen o sus proporciones), ${ }^{3}$ se complica la comparación

2 Dos comentarios son necesarios respecto a este tipo de especificación de una función de bienestar social: primero, se supone que las funciones de utilidad individual son cóncavas (la utilidad marginal es una función decreciente del ingreso), $\mathrm{y}$ segundo, suponemos que las utilidades individuales son comparables y por lo tanto agregables.

3 Esto siempre que las propensiones marginales a consumir varíen en función del ingreso. 
del producto generado bajo diferentes formas de distribución inicial (de esto se hablará más en la siguiente sección). ${ }^{4}$

\section{DistribUCIÓN DEL INGRESO Y EFICIENCIA}

El concepto de eficiencia en economía ha propiciado muchas confusiones. Existen dos posiciones extremas: 1a de aquellos economistas para los cuales la "eficiencia" es el único objetivo válido, y la de aquellos para quienes la palabra está investida de un sabor ideológico, apología de la explotación en el sistema capitalista. Por eso, antes de comenzar a discutir la relación entre eficiencia y distribución del ingreso, es conveniente aclarar el significado de este término y los límites de su utilidad.

En la teoría económica se pueden distinguir dos conceptos fundamentales de eficiencia: uno ingenieril y otro económico. El concepto de eficiencia ingenieril está implícito en la función de produción microeconómica para la firma, donde se asocia un nivel de producto máximo para cualquier combinación de insumos. La eficiencia económica se define por lo general en relación con un óptimo de Pareto, donde se determinan los precios (de insumos y productos). Como vimos en la sección anterior, los precios dependen de la distribución del ingreso inicial, por lo tanto, es posible encontrar tantos puntos de eficiencia paretiana como distribuciones iniciales se tengan. Se puede probar que cualquier óptimo paretiano es eficiente en el sentido de que no se desperdician ni recursos ni "utilidad" (niveles de satisfacción individual). E1 punto discutible radica entonces en por qué aceptar una situación que, aunque eficiente económicamente desde el punto de vista paretiano, puede provenir de una distribución "indeseable" y donde lo más probable es que a su vez genere una distribución de la misma naturaleza.

Pasemos ahora a evaluar la utilidad de los criterios paretianos para elegir entre dos alternativas distributivas. Supongamos la situación que se esquematiza en la gráfica 1 .

En este diagrama en donde $U_{x}$ y $U_{y}$ expresan la satisfacción de los individuos $x$ e y respectivamente, se presenta un número infinito de dis-

4 Antes de pasar a la siguiente sección convendría hacer mención de otro aspecto de la relación entre distribución del ingreso y bienestar. Se puede demostrar que bajo los supuestos de concavidad de la función de utilidad individual, la función de bienestar social:

$$
W(y)=\sum_{i} U\left(y_{i}\right) \text {, en donde } y \text { es un vector de ingresos; }
$$

tendrá un valor más alto a medida que la distribución sea más equitativa. Este resultado se puede generalizar a formulaciones donde no se necesita agregar funciones de utilidad individual, y hasta donde ni siquiera haga falta introducirlas de manera explícita. Para mayor detalle véase A. Sen, On Economic Inequality, Oxford University Press, 1973, pp. 49-51. 


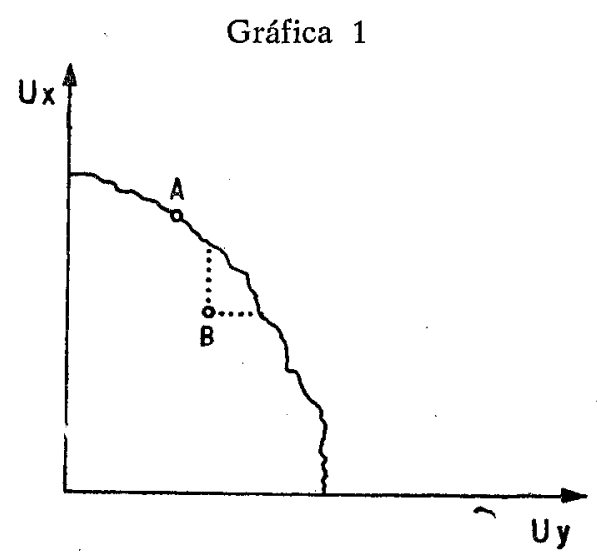

tribuciones diferentes y la combinación de utilidades individuales que corresponden a cada distribución. El criterio de "optimalidad" paretiana permite afirmar que todas las combinaciones que se encuentran sobre la frontera son eficientes, en el sentido de que no se desperdicia utilidad para ninguna de las dos personas. El punto $B$ en este sentido es ineficiente: se puede incrementar la utilidad de al menos una de las personas, sin afectar el nivel de utilidad del otro. ${ }^{5}$

Sin embargo, si se quiere comparar el punto $A$ con el punto $B$ en términos de bienestar, el criterio paretiano no puede decir nada. Si se comienza con una situación $B$ no se puede afirmar que una situación $A$ es "mejor" por ser eficiente, ya que en la distribución implícita en $A$ hay una pérdida de utilidad (bienestar) para una de las personas. Por lo tanto si, dada una distribución inicial y dadas ciertas distorsiones en el mercado, obtenemos el punto $B$ (que a la vez implica una cierta distribución resultante), no se puede afirmar que si se hacen desaparecer las distorsiones y se obtiene como resultado el punto $A$ (eficiente) y su distribución resultante correspondiente, el nivel de bienestar sea mejor en $A$ que en $B^{6}$

5 Todas las combinaciones posibles enmarcadas dentro y sobre las líneas del ángulo recto con vértice $B$, son mejores que $B$.

6 Esto conduce a otro aspecto interesante: la teoria del "segundo óptimo". Véase E. J. Mishan, "A Survey of Welfare Economics", Surveys of Economic Theory: 1. Esta teoría expresa que si existe alguna distorsión en el sistema, por ejemplo la existencia de oligopolios, que implicaría la imposibilidad de lograr el óptimo, el segundo óptimo (second best) no consiste en evitar distorsiones en otros lados, sino por el contrario, puede implicar distorsiones en otros mercados también, por ejemplo en el del trabajo. Esto es pertinente para contrarrestar el argumento de que no es conveniente introducir una ley de salarios mínimos porque produce ineficiencia en la asignación de recursos. Esto no quiere decir que la implementación de salarios mínimos no pueda producir o agravar otro tipo de efectos indeseables como el desempleo o la inflación. 
Otro argumento muy utilizado en favor o en contra de la igualdad en la distribución del ingreso, es su efecto sobre el crecimiento global del producto. Existen muchos economistas que arguyen que la igualdad disminuye la tasa de crecimiento (porque disminuye la tasa de acumulación) mientras otros opinan que la incrementa (a través del impacto sobre la demanda agregada). Los que pertenecen al primer grupo consideran entonces que una mejor distribución es "ineficiente" porque trae consigo tuna disminución en el crecimiento del producto global; los otros opinan lo contrario.

Antes de discutir este aspecto más en detalle conviene hacer ciertas consideraciones sobre la comparabilidad posible entre dos niveles de producto total. En la primera sección se dijo que la distribución inicial determina los tipos de bienes que se producen, las cantidades y sus precios; esto quiere decir que si se modifica la distribución el resultado va a ser un nuevo conjunto de bienes (pueden variar sólo las cantidades, o bien, pueden producirse bienes "nuevos") y precios. ¿Cómo podemos comparar ambos niveles de producto? He aquí de nuevo el viejo problema de los números índices: ¿qué precios usamos para valuar las nuevas cantidades: los anteriores o los posteriores a la redistribución? Es obvio que los resultados serán diferentes según el índice que se seleccione. En principio se puede decir que una comparación de los productos monetarios no tiene sentido. Sin embargo, no se puede comparar el producto real sin la ayuda de los precios: no se pueden comparar "peras con manzanas". Para hacer posible una comparación que tenga sentido se debe hacer uso de números índices para deflacionar los productos monetarios, y como ya se señaló, los resultados van a ser diferentes según el precio que se utilice para la construcción de los índices. Más aún, no se puede definir uno de los índices como más válido que el otro; entonces, lo único que queda es calcular el ingreso real con ambos índices y comparar los resultados.

Si se elimina el ineludible inconveniente que plantea la comparación, se vuelve al tema anterior: la relación (directa o inversa) que puede existir entre una distribución más equitativa y la tasa de crecimiento del producto real. A priori, las dos posiciones tienen algo de razón, pero la respuesta requiere de constatación empírica; es muy posible que ambos fenómenos ocurran, pero lo importante es verificar cuál de ellos predomina, y para determinar esto es necesario hacer el análisís con datos concretos del país en cuestión.

La posición que sostiene una relación directa entre una distribución más equitativa y la tasa de crecimiento basa su argumento en el efecto multiplicador que tenga un incremento en el nivel de consumo sobre la estructura productiva; es decir, se basa en el conocido argumento keynesiano del impacto que pueda tener un incremento de la demanda agregada sobre el producto total a consecuencia de un aumento en el nivel de consumo agregado. El incremento en el consumo como consecuencia de una distribución más equitativa se basa en el supuesto de que la propensión 
marginal a consumir es mayor a medida que el ingreso es menor, y que una política de redistribución de mayores a menores ingresos trae como consecuencia un incremento en la propensión marginal a consumir para toda la economía.

En el extremo opuesto está la postura que mantiene una relación inversa entre una distribución más equitativa y la tasa de crecimiento. Ésta se basa en cierta forma en el resultado recíproco de la postura anterior: si aumenta la propensión maginal a consumir global, y por lo tanto el consumo global, al pasar a una distribución más equitativa otro efecto posible es que disminuya el ahorro global y, por lo tanto, baje el nivel de acumulación, lo que a su vez produciría una disminución en la tasa de crecimiento.

La preocupación sobre los efectos que pueda tener una política redistributiva sobre la tasa de crecimiento, se refiere más que nada al impacto que la redistribución pueda tener sobre los niveles de consumo total y ahorro total, y sobre los efectos que los cambios en estas variables tengan sobre la tasa de crecimiento.

Cabe mencionar aquí que la disyuntiva entre niveles de consumo agregado (y por lo tanto ahorro) y la tasa de crecimiento, existe tanto en las economías de mercado como en las economías planificadas. Entre aquellos problemas a los que se enfrenta el planificador está decidir qué parte del producto se destinará al consumo presente y qué parte a inversión, o sea a consumo futuro. Una forma de resolver esto es, mediante la utilización de la técnica de programación dinámica, establecer como una de las funciones objetivo la maximización del consumo en cada período de tiempo durante todo el horizonte de planeación (por supuesto la solución variará en función del horizonte de planeación escogido).

\section{LA TEORÍA ECONÓMICA Y LA DISTRIBUCIÓN PERSONAL DEL INGRESO}

Si buscamos la explicación de la distribución personal del ingreso en la estructura analítica de la teoría económica, lo más que obtendríamos sería una buena dosis de frustración. Tanto en el esquema clásico como en el neoclásico, la teoría se centra en explicar la distribución funcional del ingreso. En la segunda sección de este trabajo hemos visto que los precios de los factores están directamente influenciados por la distribución personal inicial del ingreso, o sea que la distribución funcional que resulte del proceso productivo es una consecuencia de la distribución personal de la que se parte. $Y$ entonces surge la pregunta, ¿qué determina la distribución personal inicial? Aquí posiblemente el único enfoque teórico del que obtendríamos alguna respuesta es el de la economía política a través de su explicación histórica de cómo ciertas clases sociales y ciertos sectores dentro de las clases se apropian de la mayor parte del excedente económico en el tiempo.

Ahora bien, a cada distribución funcional que resulta del proceso pro- 
ductivo corresponde, a su vez, una nueva distribución personal. Por desgracia no existe una relación que adjudique a cada distribución funcional una personal. Es decir, no podemos encontrar una relación unívoca entre la distribución personal y la funcional; esto se debe en esencia a que la distribución del ingreso percibido por cada uno de los propietarios de los factores no es uniforme (es decir, no todos los trabajadores reciben el mismo salario, ni todos los capitalistas el mismo nivel de beneficios, ni todos los "terratenientes", urbanos y rurales, el mismo nivel de "renta"), además, por el hecho de que una misma persona recibe ingresos asociados a diferentes factores de la producción. Por lo tanto, las teorías que intentan explicar la distribución funcional del ingreso no avanzan necesariamente en la explicación de la distribución personal.

Por ejemplo, el minifundista recibe parte de su ingreso como retribución a su fuerza de trabajo ocupada en otros predios, y parte del ingreso (aunque sea en especie) lo deriva de su propio predio, o sea del factor tierra (ya que si él no la cultivara la podría rentar a otra persona).

De cualquier forma, es de utilidad investigar qué tipo de distribución personal resultaría de la distribución funcional que plantean los diferentes enfoques teóricos.

\section{Teoría clásica}

Si partimos del modelo ricardiano simple donde el ingreso se divide funcionalmente entre tres factores que corresponden al terrateniente, al capitalista y al trabajador, la posible distribución personal que resultara podría buscarse de la siguiente manera. Si suponemos que la fuerza de trabajo es homogénea y la tierra en cambio es heterogénea, o sea que existen tierras de mejor y peor calidad, y un comportamiento malthusiano de la fuerza de trabajo, el resultado sería que en el largo plazo el salario se igualaría al salario de "subsistencia", los beneficios extraordinarios desaparecerían y la tasa de ganancia sería la misma en todos los sectores de la economía, y los terratenientes obtendrían rentas diferentes dependiendo de la calidad de la tierra que poseen.

\section{Teoría marxista}

La teoría marxista coneluiría que los beneficiarios del sistema serían los capitalistas que por su control monopólico tenderían no sólo a mantener sino a aumentar su tasa de ganancias, y los trabajadores también aquí terminarían por recibir un salario de "subsistencia", definido por la cantidad de ingreso necesario para asegurar su manutención y reproducción. Los trabajadores no podrían aumentar su ingreso por encima del salario de subsistencia debido a la existencia del "ejército de reserva" de desempleados y la tecnología que contribuiría a que este proceso no se modificara (intensiva en capital) 
En el esquema marxista entonces la distribución del ingreso estaría muy concentrada: los capitalistas se volverían cada vez más ricos y los trabajadores relativamente más pobres. Más aún, la riqueza de los capitalistas se debe a la apropiación indebida de lo que correspondería al trabajador, ya que en el esquema de la teoría objetiva del valor lo único capaz de generar valor es el trabajo y al trabajo sólo se le paga en valor lo necesario para su producción y reproducción.

Sin embargo, si dejáramos que la distribución del ingreso se determinara en función de la teoría objetiva del valor, obtendríamos una distribución desigual siempre que la fuerza de trabajo no fuera homogénea (o sea que existieran diferentes calidades de mano de obra). Esto se debería simplemente a que lo que se le pagara al trabajador calificado por hora de trabajo sería mayor de lo que recibiera el no calificado, y mientras existiera una distribución concentrada en las calificaciones la distribución del ingreso correspondiente también sería concentrada.

\section{TEORÍAS DE LA DistribUCIóN PERSONAL DEL, INGRESO}

Esta falta de efectividad de la teoría económica para explicar la distribución personal implica la necesidad de buscar otras alternativas. En principio se puede decir que existen dos formas de abordar el problema: una, que comienza con algunos supuestos apriorísticos del comportamiento humano y de allí infiere la distribución del ingreso resultante del mismo. La otra, parte de la evidencia empírica y trata de encontrar el compotamiento que produciría una distribución como ésa. Este último tipo de enfoque, a su vez, se puede subdividir en dos tipos de análisis: la explicación a través de factores económicos y sociales únicamente, y aqueJla basada sólo en procesos estocásticos.

Existen tres clases de distribuciones probabilísticas que se han vincue lado a la distribución del ingreso: la distribución de Pareto, la normal y la lognormal. La distribución de Pareto ${ }^{7}$ se supone que obedecería a un comportamiento donde la disminución relativa en el número de personas a medida que el ingreso crece es cada vez más pequeña y disminuye en proporción al ingreso. Pareto encontró que esta distribución daba un ajuste casi perfecto para el comportamiento del ingreso en algunos países europeos en el siglo pasado y por lo tanto creyó haber descubierto que la distribución del ingreso era constante independientemente del país y del tiempo (he ahí el por qué se le llama la "ley de Pareto"). Sin embargo, estudios posteriores demostraron que en realidad el ajuste de esta distribución sólo era bueno cuando se aplicaba al rango de altos ingresos.

Aquellos que sostienen que la distibución del ingreso es normal se

7 Esta distribución presenta la siguiente forma: $N=A X^{-a}$ donde $N$ es el número de ingresos que excede tin cierto nivel de ingreso $X$, y $A$ y $a$ son constantes. 
basan en el supuesto de que las "aptitudes" de las personas, en particular las características físicas, presentan una distribución aproximadamente normal, y por lo tanto sería de esperarse una distribución normal del ingreso. Los estudios empíricos han demostrado que la distribución global del ingreso nunca presenta una forma normal, pero a medida que se va separando a los perceptores de ingreso en categorías más homogéneas, la distribución dentro de estos grupos va perdiendo su asimetría. Si aplicáramos a la distribución normal una explicación de procesos estocásticos esto implicaría que la oportunidad de incrementar el ingreso en una cierta cantidad absoluta es la misma para todos los niveles de ingreso, lo cual es poco realista.

La distribución lognormal implica que la distribución del logaritmo del ingreso presenta una forma normal, pero la distribución del ingreso presenta una cola más larga hacia la derecha. El proceso estocástico implícito en esta forma de distribución es que la probabilidad de incrementar el ingreso en una cierta proporción es independiente del nivel de ingreso. La distribución del ingreso global cuando se ha estimado para distintos países muestra un comportamiento más parecido a la distribución lognormal que a la normal. Sin embargo, se observa que el ajuste en este caso tampoco es perfecto y esto posiblemente se deba a que la probabilidad de incrementar el ingreso en una cierta proporción tampoco es independiente del nivel de ingreso. Por ejemplo, la probabilidad de que un rico pueda aumentar su ingreso en un $10 \%$ no es la misma que para un pobre, y es de esperarse que la probabilidad del primero será, en general, mayor que la del segundo.

Ahora bien, si se tratara de encontrar una teoría que explicara la distribución perśonal del ingreso ¿por dónde comenzar? En principio se puede decir que el ingreso es lo que las personas reciben como pago por el uso que hacen otras personas de las "aptitudes" que cada individuo posee. Por lo tanto, la distribución personal del ingreso dependerá de la distribución de estas "aptitudes" y del valor que se les asigne. Siguiendo la clasificación de Champernowne ${ }^{8}$ estas "aptitudes" se pueden dividir en cinco tipos principales: $i$ ) la posesión de habilidades; ii) la posesión de capital; iii) la posesión de tierra; iv) la posesión de ventajas provenientes de condiciones monopólicas, y v) algo que se podría denominar la posesión de "buena suerte".

Estas "aptitudes" a su vez pueden reclasificarse en tres grupos:

1) Las "aptitudes" que no se pueden obtener a través del mercado, como son las habilidades innatas y la "buena suerte";

2) Las "aptitudes" que se pueden adquirir en el mercado pero que no se pueden producir, como son la tierra y las ventajas monopólicas

8 D. G. Champernowne, The Distribution of Income Between Persons, Cambridge University Press, 1973. 
otorgadas por la posesión de algún bien que no se puede reproducir (obras de arte, paisajes, etc.) o por el acceso a información;

3) Las "aptitudes" que se pueden obtener en el mercado y que además se pueden producir como son el capital, la habilidad adquirida a través del entrenamiento o la educación, y las ventajas monopólicas que son vendibles como pueden ser derechos y patentes, o aquellas que no son sostenibles en el largo plazo como el uso de ciertas tecnologías adquiribles por otras firmas.

A su vez, las influencias que afectan los valores relativos de estos atributos se pueden resumir de la siguiente forma: ${ }^{9}$

Clase de "atributo"

1) Habilidad

2) Capital

3) Tierra

4) Ventajas de monopolio

\section{Influencias principales}

Población, ambiente y herencia; condiciones climáticas y de salubridad, el nivel de vida; facilidades educativas; movilidad social; la demanda de habilidades; política sindical; etc.

Cantidad y tipo de acumulación realizada en el pasado, la cual depende de:

población y dotación de tierras y otros recursos naturales;

leyes de la propiedad;

período de industrialización;

capacidad y voluntad de ahorro de la pobla. ción;

desigualdad en la distribución del capital; política fiscal y de inversión del estado; política bancaria (crediticia); etc.

Dotación disponible de tierra bajo y fuera de cultivo; demanda por el uso de la tierra; oportunidades y nivel del comercio internacional; etcétera.

Medios de comunicación entre personas, distribución de bienes $\mathrm{y}$ acceso a información; restricciones legales; economías de escala; influencias del sector empresarial en ámbito político; etc.

La tesis planteada por Champernowne con respecto al comportamiento de la distribución en el tiempo es que, bajo lo que él llama "fuerzas de cambio" (por ejemplo una estructura impositiva que no varía) constantes, la distribución del ingreso tiende a una cierta distribución de equilibrio, independientemente de cuál fuera la distribución personal del ingreso inicial. El argumento detrás de esta tesis es el siguiente: la canti-

9 Ibid., p. 71. 
dad de una cierta "aptitud" que una persona posee en un momento dado en el tiempo depende de la cantidad de esta "aptitud" que poseía en ei año anterior y la cantidad adquirida o perdida desde entonces. Sin embargo, como hay ciertas "aptitudes" que son intercambiables por otras, la variable relevante es entonces el valor agregado de las "aptitudes". Por lo tanto, la tesis sería que el valor agregado de las "aptitudes" de hoy depende del valor agregado de las mismas en el año anterior y de los cambios ocurridos en este valor desde entonces. El mismo argumento se aplica a la continuidad que puede haber entre el valor agregado de "aptitudes" del padre y el de su hijo. El objetivo es encontrar cuáles son las fuerzas que determinan la distribución del ingreso, o sea la distribución del valor agregado de "aptitudes" en el tiempo. Es decir las "fuerzas de cambio" que intervienen para causar las modificaciones en el ingreso, o sea, en la posesión de "aptitudes" para obtener ingreso por parte de los diferentes individuos en el tiempo. Champernowne comprueba que si las "fuerzas de cambio" son constantes o se modifican suavemente la distribución del ingreso tiende a una distribución de equilibrio.

La ventaja de abordar el estudio de la distribución de esta manera es que con la ayuda de una computadora y a partin de una cierta distribución, se puede estimar cuál sería la distribución de equilibrio a la que se llegaría y el tiempo que se tardaría en lograr este equilibrio, si se suponen constantes ias "fuerzas de cambio". De la misma manera, con técnicas de simulación, se puede estimar la distribución de equilibrio que se obtendría en el caso de modificarse una o varias de las "fuerzas de cambio". Por ejemplo, qué sucedería si el crecimiento de la población de ingresos bajos disminuyera en una cierta proporción; o qué pasaría si se aboliera la propiedad privada del capital y se permitiera que cada miembro de la sociedad recibiera por igual los ingresos derivados del uso de capital; o qué pasaría si se modifica el sistema impositivo; etc. ${ }^{10}$

Este enfoque contrasta con aquellos que intentan encontrar una explicación de la estructura de la distribución personal del ingreso mediante el método de descomposición de la varianza, como la teoría de la inlormación desarrollada por Theil; ${ }^{11}$ o de la descomposición del índice de Gini. ${ }^{12}$ El método de la descomposición de la varianza consiste en determinar en qué medida los diferentes factores que intervienen en la determinación del ingreso contribuyen a la desigualdad global; en otras palabras, cuáles "aptitudes" son más "responsables" de la conformación de la distribución del ingreso. Por ejemplo, se podría determinar en cuánto contribuyen a la desigualdad las disparidades en los niveles de educa-

10 Esto se lograría al introducir nuevos valores a las probabilidades de la matriz de transición. Para la persona interesada en la confección de dicha matriz de transición véase Champernowne, ibídem, p. 194.

$11 \mathrm{H}$. Theil, Economic and Information Theory, Amsterdam, 1967.

12 J. Fei y G. Ranis, "Income Inequality by Additive Factor Component", Center Discussion Paper No 207, Economic Growth Center, Yale University, junio de 1974. 
ción (que equivale a la "aptitud" habilidad adquirida), o la concentración de la propiedad de la tierra o el capital. ${ }^{13}$

En principio este tipo de análisis aplicado a casos empíricos daría la información necesaria para saber dónde se encuentran los orígenes de la desigualdad y permitiría implementar políticas destinadas a igualar la distribución. En realidad, la implementación de este tipo de políticas, si éstas se mantienen en el tiempo, equivaldría a modificar la matriz de transición de procesos markovianos planteada por Champernowne. Es decir, que si se logra estimar con cierta precisión la matriz de transición, y con la ayuda de los métodos de descomposición de la varianza se encuentra la preponderancia y dirección con que los diferentes factores afectan la distribución, se podría comprobar si en realidad las políticas destinadas a combatir estos efectos concentradores llevarán, en el largo plazo, a una distribución esperada.

\section{BIBLIOGRAFIA}

M. Bronfenbrenner, Income Distribution Theory, Chicago, Aldine-Atherton, 1971.

D. F. Champernowne, The Distribution of Income Between Persons, Cambridge University Press., 1973.

F. Bator, "The Simple Analytics of Welfare Maximization", American Economic Review, marzo de 1957.

J. Fei y G. Ranis, "Income Inequality by Additive Factor Components", Economic Growth Center, Yale University, junio de 1974.

A. García, "La distribución del ingreso y empleo en México", Demografía Y ECoNomía, Vol. VIII, Núm. 2(23), 1974, pp. 115-140.

H. Lydall, The Structure of Earnings, Oxford, The Clarendon Press, 1968.

A. Fishlow, "Brazilian Size Distribution of Income", American Economic Review, mayo de 1972.

D. Keesing, "Causes and Implications of Growing Inequality of Income within Developing Countries", Research Center in Economic Growth, Stanford University, febrero de 1972 .

E. J. Mishan, "A Survey of Welfare Economics", Surveys of Economic Theory: 1.

T. Negishi, "Welfare Economics and Existence of a Equilibrium for a Competitive Economy", Universidad de Tokyo.

A. Sen, On Economic Inequality, Oxford University Press., 1973.

W. Van Ginneken, "Análisis de descomposición del índice de Theil aplicado a la distribución del ingreso familiar en México", DEMografíA Y ECoNomía, Vol. IX, Núm. 1(25), 1975, pp. 93-112.

13 Un estudio que aplica la metodología de la teoría de la información para el caso de México es el de Von Ginneken, "Análisis de descomposición del índice de Theil aplicado a la distribución del ingreso familiar en México, DemoGRAFí́ Y ECONOMía, Vol. IX, Núm. 1. 